\title{
Object Based Image Segmentation Algorithm of SAGA GIS for Detecting Urban Spaces in Yaoundé, Cameroon
}

\author{
Polina Lemenkova \\ Schmidt Institute of Physics of the Earth, Russian Academy of Sciences. Laboratory of Regional Geophysics and \\ Natural Disasters (Nr. 303). Bolshaya Gruzinskaya St, 10, Bld. 1, Moscow, 123995, Russia; \\ email: pauline.lemenkova@gmail.com \\ Received: 7 October 2020; Revised: 7 December 2020; Accepted: 10 December 2020; \\ Published online: 12 December 2020
}

\begin{abstract}
Present study is focused on the satellite image processing by means of SAGA GIS. The objective of the study is assessment and analysis of the core urban areas and its spatial distribution in the limits of the city and suburbs. The study area includes Yaoundé and its surroundings, Republic of Cameroon. The methodology includes Object Based Image Segmentation (OBIS) approach by SAGA GIS. The paper presents a methodologically structured workflow used in SAGA GIS for segmentation of the Sentinel-2A image. The segmentation techniques includes adjusting technical parameters, performing neighborhood approach and post-processing procedures (unsupervised classification, number of clusters). The OBIS model and SAGA GIS were used as main methods and machine learning techniques for image segmentation. Data include Sentinel-2A satellite image with high resolution $(10 \mathrm{~m})$. The image was analyzed by two approaches of cell neighborhood analysis: Moore and Neumann. The results showed following numerical parameters of the computed area: the perimeter of 1,060,560 km and an area estimated for the Yaoundé city 191,745,000 $\mathrm{km}^{2}$. The Neumann approach demonstrated better results for image clustering. The results presented automatically detected and separated segments of the city areas and other land cover types (savannah, forests, mountains). The spectral reflectance of various land cover types on a satellite image enables to group pixels of the image into classes using segmentation technique which has an important impact on the conceptual methodology of the urban mapping. The results of the image segmentation show the average values of the Neumann approach more correct in urban area than Moore approach. The accuracy assessment demonstrated $74.63 \%$ for the core urban area by using the Neumann method. The applicability of SAGA GIS for automated methods of image processing using machine learning algorithm of OBIS is presented and the advantages are discussed. The study demonstrated the effectiveness of the high-resolution Sentinel-2A for socio-economic studies, exemplified by urban mapping where remote sensing data serve as reliable sources of geoinformation. The advantages of the OBIS are discussed with detailed explanation of the SAGA GIS workflow.
\end{abstract}

Key words: Sentinel-2A, object based image analysis, segmentation, machine learning, SAGA GIS

Citation: Lemenkova, P. (2020). Object Based Image Segmentation Algorithm of SAGA GIS for Detecting Urban Spaces in Yaoundé, Cameroon. Central European Journal of Geography and Sustainable Development, 2(2), 3851. https://doi.org/10.47246/CEJGSD.2020.2.2.4

https://doi.org/10.47246/CEJGSD.2020.2.2.4

\section{| Full text |}

